\title{
3D piezoresistive silicon microprobes with stacked suspensions for tailored mechanical anisotropies
}

\author{
D. Metz, N. Ferreira, A. Dietzel \\ Technische Universität Braunschweig, Institute of Microtechnology \\ Braunschweig, Germany \\ d.metz@tu-braunschweig.de
}

\begin{abstract}
Different kinds of piezoresistive microprobes based on silicon have been developed to enable measurement with high accuracies. However, the typical mechanical anisotropy of such systems leads to the slip of the tip, when probing inclined surfaces. Here, a novel microprobe design is presented, which can be tailored to provide a range of anisotropy or even a perfect isotropy. In the first approach, the microprobe is composed of two stacked silicon membranes. In the second approach, a stainless steel suspension in the form of a laser structured foil is stacked on a silicon membrane. Geometrical parameter studies were carried out by mechanical FEM simulations to determine their influence on the stiffnesses in all spatial directions and to predict anisotropies. Microsystems with selected geometries were fabricated and stacking was obtained through selective adhesive transfer and bonding on a wafer level. Prototypes with anisotropies between 3 and 0.4 were characterized confirming the simulations.
\end{abstract}

Keywords - 3D micro probing system, piezo-resistive effect, tactile coordinate measurement, laser structuring, wafer-level bonding and tailored mechanical anisotropy.

\section{INTRODUCTION}

The needs and challenges of measuring complex microstructures are growing. Two fundamentally different types of sensors for measuring microstructures are widely employed in coordinate measurement machines (CMMs). On one hand, optical sensors are used, which enable a fast probing of structures with many measuring points. On the other hand, tactile sensors enable an accurate measurement with the possibility of probing structures which are hidden and optically not accessible. Microprobes are typically still integrated into specific, accurate and expensive $\mu \mathrm{CMMs}$. However, measuring small structures with a high accuracy on more widely available conventional CMMs through microprobes that can be integrated is an advantageous alternative [1, 2]. A tactile microprobe is typically a sensing device with an attached stylus. The tip of the stylus comes in contact with the measured surface, and the sensing device registers the displacement of the tip. Tactile micro probing systems on the base of different physical principles have been developed and optimized [3, 4]. Next to sensing properties and small tip diameters, the mechanical construction of the suspension carrying the stylus is also important. These miniaturized suspensions typically entail anisotropic mechanic stiffnesses, which can lead to slipping of the tip when probing inclined surfaces. This increases measurement uncertainty.

A vibrating "non-contact" silicon 3D-microprobe has been developed where the stylus is suspended with three silicon springs. During probing, the oscillation drift can be accurately measured [5]. In this case, the mechanical anisotropy is essential in getting different resonance frequencies in each direction. A combined optical/tactile microprobe was developed where a fiberglass with a melted tip is used as a stylus, of which the position is captured through the reflection of laser light from the tip [6]. Through an optimized leaf spring, an anisotropy of 1.4:1 could be achieved [7]. This microprobe is only available on the CMM from Werth company [8]. Further, a precision machined micro probing system has been fabricated from an aluminium cube, which is composed of a three parallelogram mechanism with elastic hinges. This allowed a perfect isotropic mechanical stiffness of $20 \mathrm{mN} \cdot \mathrm{mm}^{-1}$ to be achieved [9]. However, the inertial mass of this microprobe combined with its low stiffness prohibits its integration in a conventional CMM because the low resonance frequency renders movements of the probing system impossible. Previous works include a probing system with a variable stiffness [10], which is able to achieve close to isotropic mechanical behavior (1.3) by using a special suspension structure and applying piezo-electric compressive loads. Furthermore, three-legged suspension structures for low-probing forces have been also investigated [11]. These flexures were made from $50 \mu \mathrm{m}$ thin beryllium-copper sheets. The stiffness of these suspensions containing three capacitance transducers is isotropic in the main probe directions $\mathrm{X}-\mathrm{Y}$ with tolerances of about $10 \%$. These suspensions joined with a stylus having a probe sphere of about $70 \mu \mathrm{m}$ in diameter are commercially available (IBS Precision Engineering) for specific CMMs [12]. A microprobe suspended using three silicon slender rods, each with metallic piezo resistive strain gauges in a Wheatstone bridge configuration was developed, thus enabling accurate measurements [13]. An optimization by rods of different stiffnesses revealed systems isotropic in $\mathrm{X}-\mathrm{Y}$ but still anisotropic $(6: 1)$ with respect to $z$-direction [14].

In previous works [1], a silicon membrane based microprobe (Figure 1) was developed. In the middle of the membrane, a boss structure is located, on which a tungsten carbide stylus with probe ball diameters between $50 \mu \mathrm{m}$ and $300 \mu \mathrm{m}$ is mounted (Figure 1a.). Strain sensors are provided by 
piezoresistive paths realized by local diffusion doping of the membrane (Figure 1b.). Such microprobes have already been integrated into a commercial CMM (gear measuring machine P40, Klingelnberg) and a micro gear artifact has been measured for test purposes $[2,15]$. However, it was noticed that the stiffness of these systems in the $\mathrm{z}$-direction (about $20 \mathrm{~N} \cdot \mathrm{mm}^{-1}$ ) is 20 to 40 times higher than in $\mathrm{x}$-, $\mathrm{y}$-directions (about $1 \mathrm{~N} \cdot \mathrm{mm}^{-1}$ ), which leads to recognizable slipping effects. When probing a sphere with a diameter of $2 \mathrm{~mm}$, a slip up to $30 \mu \mathrm{m}$ is observed at a nominal deflection of $20 \mu \mathrm{m}$ in the sphere radius direction.

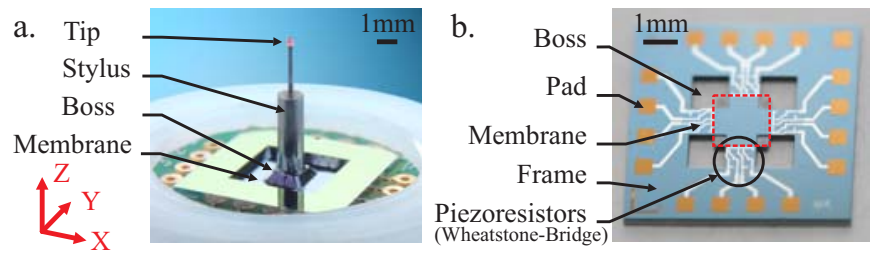

Figure 1 - a. Microprobe with full membrane and mounted stylus and b. cross-membrane-chip with the four Wheatstonebridges as described in [1].

Two stacked membranes (silicon-silicon) have already been proposed earlier to reduce the anisotropy [16], but with the compromise that the stiffnesses in all directions strongly increase. Here we present new microprobes with reduced anisotropy and even with almost perfect isotropy which allow lower $\mathrm{x}-, \mathrm{y}$-stiffness. Two stacked membrane designs (silicon-silicon and metal-silicon) will be described and the choice of design parameters will be supported by simulations. In a second part, the manufacturing and assembly process of both designs will be explained in detail. Finally, the mechanical and electrical characterization of the manufactured prototypes will be discussed in the light of the simulations.

\section{MICROPROBES WITH STACKED SUSPENSIONS}

Two new microprobe designs based on a silicon single-crossmembrane suspension (in following: single Si suspension) with piezo-resistors as sensing elements stacked with an additional mechanical suspension have been investigated. This stacked additional suspension has only a minor influence on the stiffness in the z-direction but strongly influences the stiffness in the $\mathrm{x}$ - and $\mathrm{y}$-directions, thus reducing anisotropy. In one design, a femtosecond-laser structured stainless steel foil is mounted on top of a single $\mathrm{Si}$ suspension (in following: steel/Si suspension) (Figure 2a.). In the second design, a second silicon cross-membrane is mounted on top of the first one (in following: double $\mathrm{Si}$ suspension) (Figure 2b.). The fabrication and stacking process of both substrates will be discussed later.

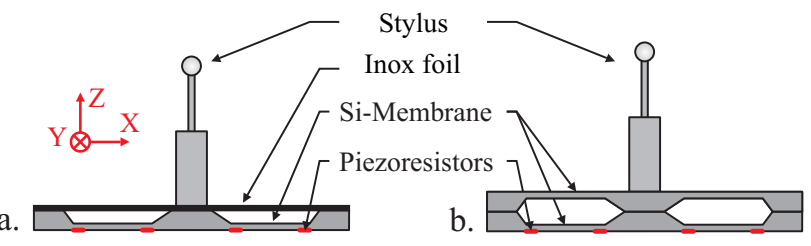

Figure 2 - New microprobe designs with stacked suspensions: $a$. steel/Si- and b. double Si suspension.

Laser machining of the metal foil allows a free definition of the geometry of this part of the suspension. In Figure 3, three different exemplary designs of steel foil suspensions are presented. The stiffnesses in the $x-y$ plane obtained from FEM-simulations of steel/Si suspension reveal that only the four-rod design with double axes symmetry is isotropic in the $x-y$ directions (Figure 3d). For this reason, only this design of foil has been considered in the following.

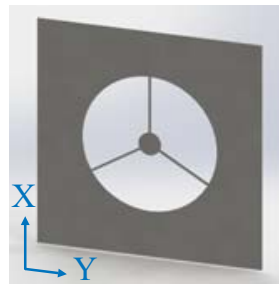

a.

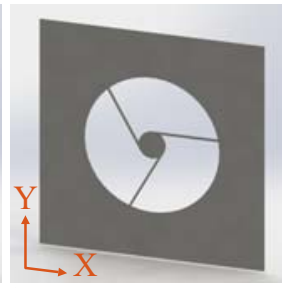

b.

Stiffness in XY plane

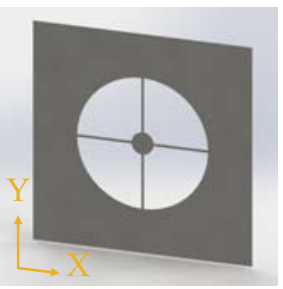

c.

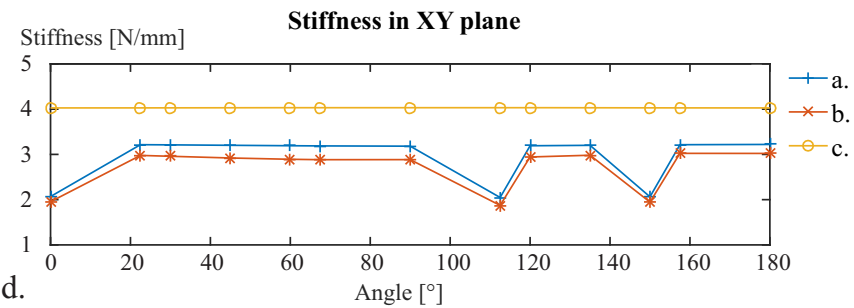

Figure 3 - Three various geometries (a., b., c.) for the stainless steel foil forming part of the mechanical suspension and $d$. the corresponding stiffnesses in the $x-y$ plane in dependence of angular direction as obtained from FEM simulation of steel/Si suspensions.

In order to get systems with tailored 3D anisotropies, geometry parameters of both the Si-membrane and the stainless steel foil can be adapted while keeping an external dimension of $6.5 \times 6.5 \mathrm{~mm}^{2}$ for the silicon chip. This is identical to previous designs and allows the use of the established concept of integration into the CMM.

\section{SimUlations}

\section{a. Simulation methods}

Static mechanical simulations (using ANSYS workbench) of both new stacked microprobe designs were undertaken, allowing comparison even with previous non-stacked designs. Material properties (Young's moduli E and Poisson ratios v) were assumed as $\mathrm{E}=193 \mathrm{GPa}[17, \mathrm{p} .360]$ and $v=0.28[18$, p. 913] for stainless steel (metal foil material, $\mathrm{X} 5 \mathrm{CrNi1} 18-10$ or AISI 304), and $\mathrm{E}=620 \mathrm{GPa}$ and $v=0.18$ for tungsten carbide (stylus material) [19, p. 114]. The anisotropic mechanical behavior of (100) silicon can be 
described by the following stiffness matrix [20, 21], where the $x-, y-$ and $z$-axis of the FEM model are aligned to the $<011>,<0 \overline{1} 1>,<100>$ directions of the silicon wafer respectively:

$$
\left(\begin{array}{ccccccc}
194.45 & & & & & \\
35.25 & 194.45 & & & & \\
63.9 & 63.9 & 165.8 & & & \\
0 & 0 & 0 & 79.6 & & \\
0 & 0 & 0 & 0 & 79.6 & \\
0 & 0 & 0 & 0 & 0 & 51
\end{array}\right)_{\mathrm{GPa}}
$$

In all simulations, the frame contour of the silicon membrane is fixed, and an external $F=\left(F_{x}, F_{y}, F_{z}\right)$ acts on the center of the tip sphere (Figure 4). Simulations reveal the resulting displacement vectors of the tip $\left(d_{x}, d_{y}, d_{z}\right)$ and the stiffness of the microprobe $\left(S_{x}, S_{y}, S_{z}\right)$ can be determined as $S_{i}=\frac{F_{i}}{d_{i}}$. The model has been meshed with the help of the proximity size function, which optimizes the size of tetraeder-elements in dependence of geometric structure width (the following settings are used: Relevance "100", Relevance Center "Coarse", Initial Size Seed "Active Assembly", Smoothing "medium", Transition "Fast", Span Angle Center "Coarse" and Num. Cell Across Gap "2"). For more than two cells over the gap the FEM results did not change. For a minimum of two cells the thinnest geometries were modeled with a finer mesh. Figure 4 also illustrates the fine mesh over Si-beams and the thin metal rods.

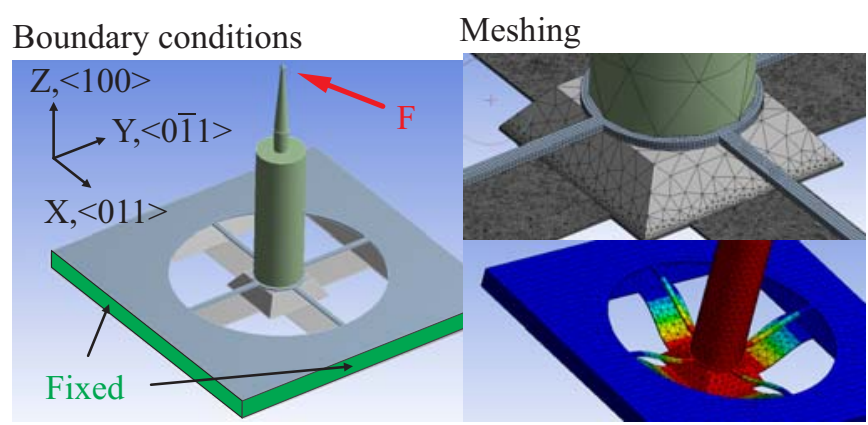

Deflection in Z-direction

Figure 4 - The model of stacked microprobe with an illustration of boundary conditions (fixed frame and force acting on the center of tip sphere) as used in the simulations. The expanded view shows the meshing of the rods and the Simembrane. The resulting deflection for a force in the $\mathrm{z}$ direction is also shown.

\section{b. Parameter study}

The geometry of the design was parameterized so that a number of variations could be automatically simulated through a variation-matrix. For each variation, the stiffness components $S_{x}$ and $S_{z}$ were determined for a force of $50 \mathrm{mN}$ as well as the mechanical anisotropy $A=\frac{S_{z}}{S_{x}}$ for futher analysis. The stiffnesses $S_{x}$ and $S_{y}$ are equal as a result of the symmetry of the system. For the suspension part made from the stainless steel foil, three geometry parameters were varied: the diameter of the suspension $d_{f o i l}$, the width of the rods $w_{\text {foil }}$ and the thickness of the foil $t_{\text {foil }}$ (Figure 5a.). Concerning the silicon cross-membrane, the thickness of the membrane $t_{m e m}$, the width of the membrane $w_{\text {mem }}$ and the width of the cross beams $w_{\text {cross }}$ (Figure $5 b$.) were varied. Finally, different styli have been investigated by reducing the tip diameter $d_{\text {tip }}$ and shaft diameter $d_{\text {shaft }}$ (Figure 5c.). The length of the stylus was fixed to $5 \mathrm{~mm}$.
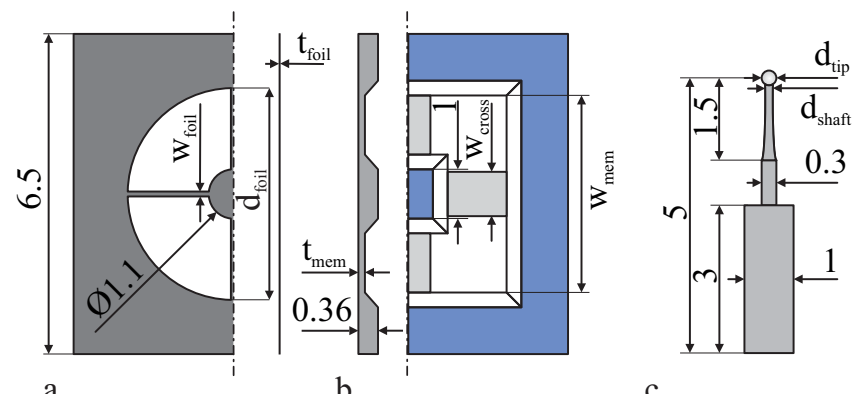

c.

Figure 5 - Geometrical parameters a. for suspension parts made of stainless steel foil, $b$. for the silicon cross-membrane and $c$. for the stylus.

\section{Stiffness of the stylus}

Four different styli have been considered according to Table 1. The radial stiffness $S_{r}$ and axial stiffness $S_{a}$ of them were determined for a stylus without any suspension. The stylus can be acknowledged as non-compressible in the zdirection. However, bending of the stylus in radial directions cannot be ignored because its stiffness is near to the stiffness of the single membrane suspension. In all directions, the stylus and the suspension can be handled as cascaded springs resulting in an equivalent system stiffness $S_{\text {eq }}=\frac{S_{\text {stylus }} \cdot S_{\text {suspension }}}{S_{\text {stylus }}+S_{\text {suspension }}}$. In all following simulations, this equivalent stiffness was determined and analysed using the stylus no. 2 .

Table 1 - Stiffness for different stylus dimensions.

\begin{tabular}{ccccc}
\hline No. & $\begin{array}{c}\boldsymbol{d}_{\text {tip }} \\
{[\mathbf{m m}]}\end{array}$ & $\begin{array}{c}\boldsymbol{d}_{\text {shaft }} \\
{[\mathbf{m m}]}\end{array}$ & $\begin{array}{c}\boldsymbol{S}_{\boldsymbol{a}} \\
{\left[\mathbf{N} \cdot \mathbf{m m}^{-1}\right]}\end{array}$ & $\begin{array}{c}\boldsymbol{S}_{\boldsymbol{r}} \\
{\left[\mathbf{N} \cdot \mathbf{m m}^{-1}\right]}\end{array}$ \\
\hline $\mathbf{1}$ & $\mathbf{0 . 0 5}$ & $\mathbf{0 . 0 3 5}$ & $1.43 \cdot 10^{3}$ & 2.63 \\
\hline $\mathbf{2}$ & $\mathbf{0 . 1}$ & $\mathbf{0 . 0 7}$ & $3.75 \cdot 10^{3}$ & 11.28 \\
\hline $\mathbf{3}$ & $\mathbf{0 . 2}$ & $\mathbf{0 . 1 2}$ & $7.58 \cdot 10^{3}$ & 27.72 \\
\hline $\mathbf{4}$ & $\mathbf{0 . 3}$ & $\mathbf{0 . 1 8}$ & $12.06 \cdot 10^{3}$ & 45.51 \\
\hline
\end{tabular}

\section{Stiffness of single Si suspension geometries}

First, a microprobe made of a single $\mathrm{Si}$ suspension was investigated with a focus on $t_{\text {mem }}, w_{\text {mem }}$ and $w_{\text {cross }}$. In Figure 6a., the stiffnesses $S_{x}$ and $S_{z}$ are given as a function of $w_{\text {mem }}$ for $w_{\text {cross }}=0.8 \mathrm{~mm}$. In Figure $6 \mathrm{~b}$., the resulting values of anisotropy are represented. 

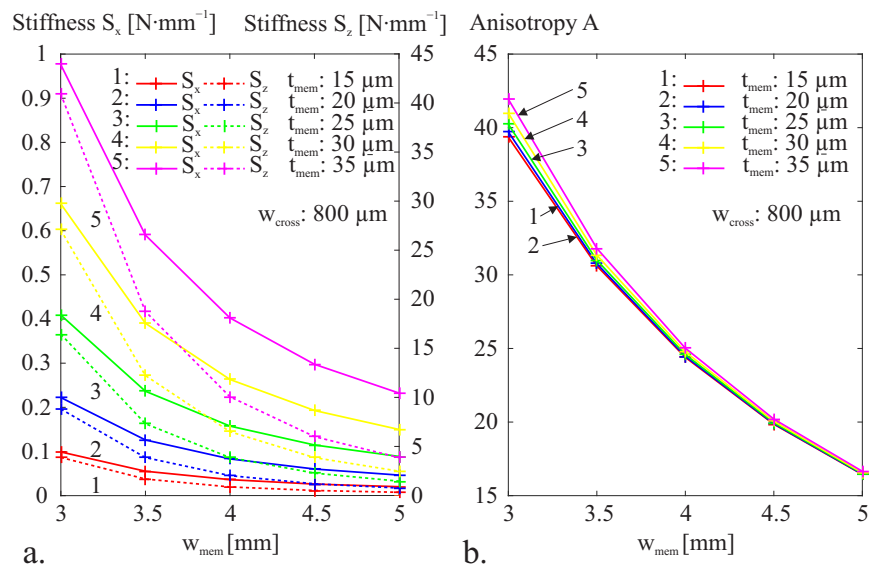

Figure 6 - a. Stiffnesses $S_{x}$ and $S_{z}$ and $b$. anisotropy A as a function of $w_{\text {mem }}$ for single Si suspension as obtained by simulations. Note: $S_{x} / S_{z}$ are plotted on different scales.

To discuss the influence of $w_{m e m}$, the data were fitted with the function $y=a \cdot w_{m e m}^{b}$ for $y=S_{x}, S_{z}$ and $A$. In Table 2, the fit results $\mathrm{a}, \mathrm{b}$ and $\mathrm{R}^{2}$ are given for the case of $t_{m e m}=25 \mu \mathrm{m}$ (curve 3 in Figure 6). To determine them a linear regression was done on the natural logarithm of $y$. Due to a higher negative b-exponent, $S_{z}$ decreases faster than $S_{x}$ with increasing $w_{m e m}$, and $A$ decreases also with $w_{m e m}$, as seen in Figure 6b.

Table 2 - Fit parameters as obtained for the influence of Simembrane geometries for the case of a single Si suspension.

\begin{tabular}{|c|c|c|c|}
\hline \multicolumn{4}{|c|}{ Influence of $\boldsymbol{w}_{\boldsymbol{m e m}}$ for $t_{m e m}=25 \mu \mathrm{m}$ and $w_{\text {cross }}=0.8 \mathrm{~mm}$} \\
\hline & $S_{x}=a \cdot w_{m e m}^{b}$ & $S_{z}=a \cdot w_{m e m}^{b}$ & $A=a \cdot w_{m e m}^{b}$ \\
\hline $\mathbf{a}$ & 10.4 & $2.87 \cdot 10^{3}$ & $2.75 \cdot 10^{2}$ \\
\hline b & -2.99 & -4.74 & -1.74 \\
\hline $\mathbf{R}^{2}$ & 0.994 & 0.998 & 1.00 \\
\hline \multicolumn{4}{|c|}{ Influence of $\boldsymbol{t}_{\boldsymbol{m e m}}$ for $w_{\text {mem }}=4 \mathrm{~mm}$ and $w_{\text {cross }}=0.8 \mathrm{~mm}$} \\
\hline & $S_{x}=a \cdot t_{m e m}^{b}$ & $S_{z}=a \cdot t_{m e m}^{b}$ & $A=c$ \\
\hline $\mathbf{a}$ & $5.72 \cdot 10^{3}$ & $1.57 \cdot 10^{5}$ & - \\
\hline b & 2.85 & 2.88 & - \\
\hline $\mathbf{R}^{2}$ & 1.00 & 1.00 & - \\
\hline c & - & - & $24.6 \pm 0.3$ \\
\hline
\end{tabular}

In Figure 7a., $S_{x}$ and $S_{z}$ are displayed as a function of $t_{m e m}$. In a similar way for $y=S_{x}$ and $S_{z}$, the function $y=a \cdot t_{m e m}^{b}$ was fitted to the simulated values and a- and b-coefficients (Table 2) are given for the case of $w_{\text {mem }}=4 \mathrm{~mm}$ (curve 3 in Figure 7a.). The parameter $\mathrm{b}$ is the same for both stiffnesses. As a consequence, the anisotropy $\mathrm{A}$ is practically not influenced by $t_{m e m}$, and for each $w_{\text {mem }}$ it can be considered as constant (Table 2). In Figure $7 \mathrm{~b}$., the stiffnesses are given as a function of $w_{\text {cross }}$. $W_{\text {cross }}$ influences $S_{x}$ and $S_{z}$ in a linear manner, and the influence of $w_{\text {cross }}$ increases with smaller $t_{m e m}$. Since both stiffnesses $S_{x}$ and $S_{z}$ linearly depend on $w_{\text {cross }}$, the anisotropy does not depend on $w_{\text {cross }}$. As example, stiffnesses $S_{x}=0.198 \cdot w_{\text {cross }}$ and $S_{z}=4.83 \cdot w_{\text {cross }}$ are obtained by simulation resulting in an anisotropy of 24.5 , for the curve 3 in Figure $7 b$.
Stiffness $\mathrm{S}_{\mathrm{x}}\left[\mathrm{N} \cdot \mathrm{mm}^{-1}\right] \quad$ Stiffness $\mathrm{S}_{z}\left[\mathrm{~N} \cdot \mathrm{mm}^{-1}\right] \quad$ Stiffness $\mathrm{S}_{\mathrm{x}}\left[\mathrm{N} \cdot \mathrm{mm}^{-1}\right]$ Stiffness $\mathrm{S}_{z}\left[\mathrm{~N} \cdot \mathrm{mm}^{-1}\right.$
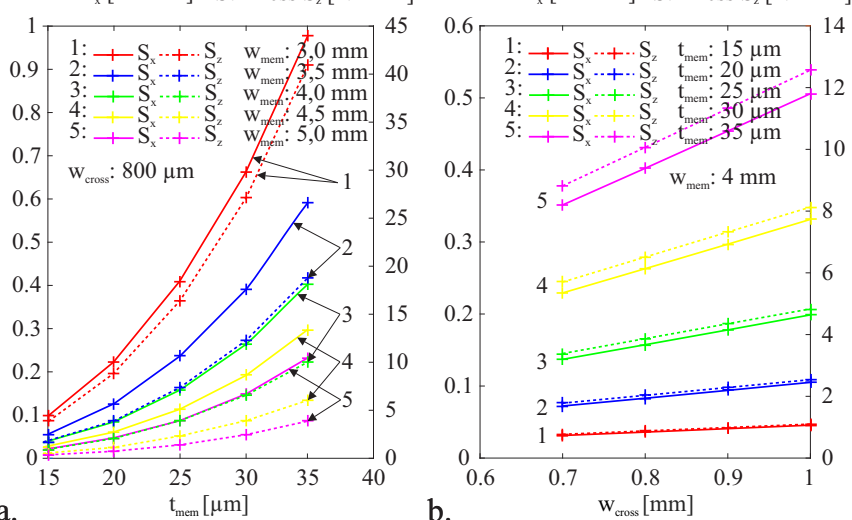

Figure 7 - Stiffnesses in $x-z$ directions as a function of $a$. $t_{\text {mem }}$ and $b . w_{\text {cross }}$ for single Si suspension as obtained by simulations. Note: $S_{x} / S_{z}$ are plotted on different scales.

The three geometry parameters of the cross membrane suspension differ in their influence on the stiffnesses of the system and can be sorted in order of strength of influence as $w_{\text {mem }}, t_{\text {mem }}$ and $w_{\text {cross. }}$. The approximated scaling of stiffnesses as $S_{x / z} \propto t_{m e m}^{3}$ and $S_{x / z} \propto w_{\text {cross }}$ can be understood when considering bending theory for a two side fixed beam [18, p. 909] which gives the stiffnesses parallel (x-direction) and perpendicular (z-direction) to the beam of length $l$, width $w$, and thickness $t$ when the load acts on a stylus of length $l_{t}$ mounted on the middle of the beam :

$$
S_{x}=\frac{12 \cdot E \cdot I}{l_{t} \cdot l_{\text {beam }}} ; S_{z}=\frac{48 \cdot E \cdot I}{l_{\text {beam }}^{3}} \text {, with I: } \frac{w_{\text {beam }} \cdot t_{\text {beam }}^{3}}{12}
$$

However, for $S_{x} \propto w_{m e m}^{-3}$ and $S_{z} \propto w_{m e m}^{-5},\left(w_{m e m}\right.$ defines the beam length $l_{\text {beam }}$ ) the influence of the boss structure has to be taken into account. With further increasing $w_{\text {mem }}$, the boss will have less influence and $S_{x}, S_{z}$ will approach $\propto w_{\text {mem }}^{-1}$ and $\propto w_{\text {mem }}^{-3}$ dependencies. For the anisotropy $A$ only $w_{\text {mem }}$ can be considered to have a considerable influence. However, an isotropic system $(A=1)$ form by a single $\mathrm{Si}$ suspension would require a cross membrane wider than $5 \mathrm{~mm}$, which would severely constrain the geometric accessibility of workpieces with deep shapes.

\section{Stiffness of double Si suspension}

Here, the influence of the membrane parameters assuming a double Si suspension with two identical silicon membranes, as sketched in Figure 2b, was investigated. In Figure 8a., $S_{x}$ and $S_{z}$ are represented in function of $w_{\text {mem }}$, which already illustrates differences from the single membrane system. $S_{x}$ is only weakly and linearly influenced by the membrane width. In contrast, $S_{Z}$ is still strongly influenced by $w_{\text {mem }}$ according to a similar power function as for single membrane design (Table 3). As expected, the absolute values of $S_{z}$ are doubled (coefficient a is about the double) compared to the single $\mathrm{Si}$ suspension, which is a consequence of the stacking of two identical membranes. The very different scaling of stiffnesses with $\propto w_{m e m}^{-4.7}$ for $S_{z}$ and with $\propto w_{\text {mem }}$ for $S_{x}$ has the result that the anisotropy, in general, is lower and can be tuned to 
the point of isotropy $(A=1)$ (Figure $8 b$.). The analysis of the influence of $t_{\text {mem }}$ shows that it plays a bigger role in the determination of the anisotropy, as for the single $\mathrm{Si}$ suspension. This result can be confirmed with Figure $8 \mathrm{~b}$. and Table 3, where the fit results of $S_{x}, S_{z}$, and $A$ are presented. As seen for $w_{\text {mem }}, t_{\text {mem }}$ influenced $S_{x}$ weakly linear, $S_{z}$ and $A$ with a power function.
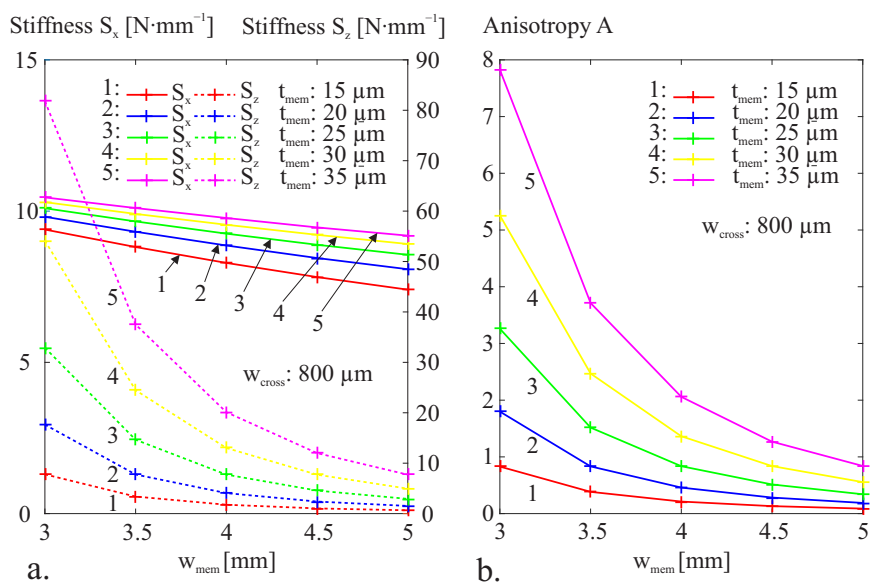

Figure $8-a$. Stiffnesses in $x-z$ direction and $b$. anisotropy $A$ as a function of $w_{\text {mem }}$ for a double Si suspension as obtained by simulations. Note: $S_{x} / S_{z}$ are plotted on different scales.

The influence of $w_{\text {cross }}$ on the stiffnesses is also linear and $A$ does not depend on $w_{\text {cross }}$ like for single Si suspensions. As example, for $t_{\text {mem }}=25 \mu \mathrm{m}$ and $w_{\text {mem }}=4 \mathrm{~mm}$, stiffnesses $S_{x}=10.9 \cdot w_{\text {cross }}$ and $S_{z}=9.67 \cdot w_{\text {cross }}$ are obtained by simulation resulting in an anisotropy of 0.9 . It should be highlighted that the linear influence of $w_{\text {cross }}$ is stronger by about 50 times for $S_{x}$ and twices as strong for $S_{z}$ when compared to single suspensions. But the influence of $w_{\text {cross }}$ on stiffnesses is weaker than the influences of $t_{m e m}$ and $w_{\text {mem }}$.

Table 3 - Fit parameters as obtained for the influence of membrane geometries for the case of a double Si suspension.

\begin{tabular}{cccc}
\hline \multicolumn{4}{c}{ Influence of $\boldsymbol{w}_{\boldsymbol{m e m}}$ for $t_{\text {mem }}=25 \mu \mathrm{m}$ and $w_{\text {cross }}=0.8 \mathrm{~mm}$} \\
\hline & $\boldsymbol{S}_{\boldsymbol{x}}=\boldsymbol{a} \cdot \boldsymbol{w}_{\boldsymbol{m e m}}+\boldsymbol{c}$ & $\boldsymbol{S}_{\boldsymbol{z}}=\boldsymbol{a} \cdot \boldsymbol{w}_{\boldsymbol{m e m}}^{\boldsymbol{b}}$ & $\boldsymbol{A}=\boldsymbol{a} \cdot \boldsymbol{w}_{\mathbf{m e m}}^{\boldsymbol{b}}$ \\
\hline $\mathbf{a}$ & $-7.70 \cdot 10^{-1}$ & $5.74 \cdot 10^{3}$ & $3.97 \cdot 10^{2}$ \\
\hline $\mathbf{b}$ & - & -4.74 & -4.41 \\
\hline $\mathbf{R}^{\mathbf{2}}$ & 0.997 & 0.998 & 0.998 \\
\hline $\mathbf{c}$ & 12.4 & - & - \\
\hline & Influence of $\boldsymbol{t}_{\boldsymbol{m e m}}$ for $w_{\text {mem }}=$ & $4 \mathrm{~mm}$ and $w_{\text {cross }}=0.8 \mathrm{~mm}$ \\
\hline & $\boldsymbol{S}_{\boldsymbol{x}}=\boldsymbol{a} \cdot \boldsymbol{t}_{\boldsymbol{m e m}}+\boldsymbol{c}$ & $\boldsymbol{S}_{\boldsymbol{x}}=\boldsymbol{a} \cdot \boldsymbol{t}_{\boldsymbol{m e m}}^{\boldsymbol{b}}$ & $\boldsymbol{A}=\boldsymbol{a} \cdot \boldsymbol{t}_{\boldsymbol{m e m}}^{\boldsymbol{b}}$ \\
\hline $\mathbf{a}$ & $7.31 \cdot 10^{1}$ & $3.13 \cdot 10^{5}$ & $1.66 \cdot 10^{4}$ \\
\hline $\mathbf{b}$ & - & 2.88 & 2.68 \\
\hline $\mathbf{R}^{\mathbf{2}}$ & 0.966 & 1.00 & 1.00 \\
\hline $\mathbf{c}$ & 7.32 & - & - \\
\hline
\end{tabular}

By stacking two cross membranes, $S_{x}$ increases and can even approach the x-stiffness of a thin stylus. This can lead to a strong deformation of the stylus and weakened sensor signals. As a further possibility to tailor mechanical properties, the top membrane width can be increased $\left(w_{m e m T}>w_{m e m B}\right)$ to reduce $S_{x}$. Simulation reveals that by increasing $w_{\text {mem }}$ of the top membrane from 4 to $5 \mathrm{~mm}$ (for $t_{m e m}=25 \mu \mathrm{m}$ and $\left.w_{\text {cross }}=0.8 \mathrm{~mm}\right) S_{x}$ reduces from 9.52 to $8.97 \mathrm{~N} \cdot \mathrm{mm}^{-1}$ and $S_{Z}$ from 8.70 to $5.81 \mathrm{~N} \cdot \mathrm{mm}^{-1}$.

\section{Stiffness of steel/Si suspension}

Assuming a steel/Si suspension composed of a metal foil part and a Si-membrane (s. Figure 2a), the influence of the geometry of the membrane was investigated while the foil dimensions were defined as $d_{\text {foil }}=w_{\text {mem }}+0.4 \mathrm{~mm}$ to allow a good overlap for assembly of both suspension parts. Further, a thickness $t_{\text {foil }}=50 \mu \mathrm{m}$ and a width $w_{\text {foil }}=0.1 \mathrm{~mm}$ were assumed. In Figure $9, S_{x}, S_{z}$ and $A$ are given as a function of width membrane $w_{m e m}$. In the stacked structure, $S_{x}$ can be considered as only weakly influenced by $w_{\text {mem }} . S_{z}$ is strongly influenced by $w_{\text {mem }}$ by about half, compared to the one for the double Si suspension, meaning that the steel/Si suspension does not or a little increase the stiffness in the z-direction. Similar as before, Table 4 gives the fitting results for the parameters $w_{m e m}$ and $t_{m e m}$.
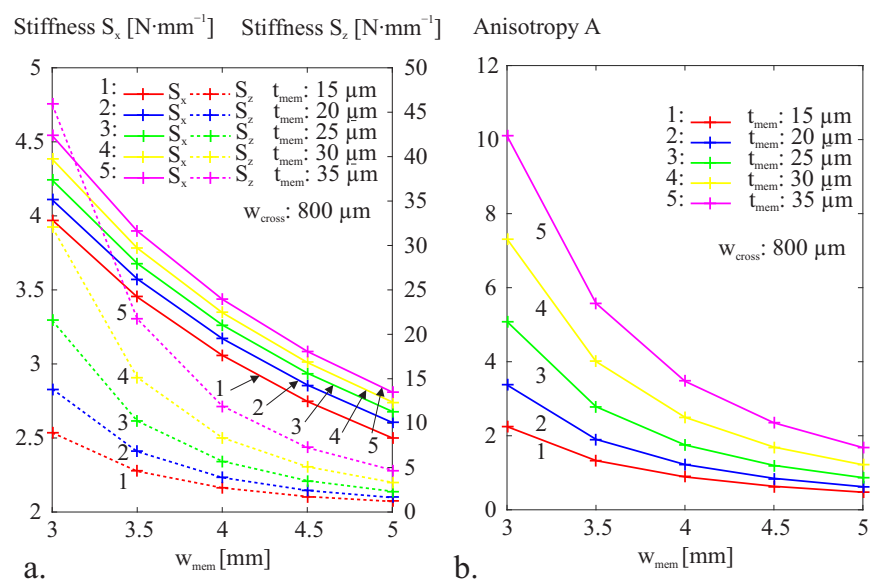

Figure $9-a$. Stiffnesses in $x-z$ directions and $b$. anisotropy $A$ as a function of $w_{\text {mem }}$ for a steel/Si suspension as obtained by simulations. Note: $S_{x} / S_{z}$ are plotted on different scales.

Table 4 - Stiffnesses and anisotropies for the steel/Si suspension as obtained by simulations. Note. Only the membrane geometries were varied.

\begin{tabular}{cccc}
\hline \multicolumn{4}{c}{ Influence of $\boldsymbol{w}_{\boldsymbol{m e m}}$ for $t_{\text {mem }}=25 \mu \mathrm{m}$ and $w_{\text {cross }}=0.8 \mathrm{~mm}$} \\
\hline & $\boldsymbol{S}_{\boldsymbol{x}}=\boldsymbol{a} \cdot \boldsymbol{w}_{\mathbf{m e m}}+\boldsymbol{c}$ & $\boldsymbol{S}_{\boldsymbol{z}}=\boldsymbol{a} \cdot \boldsymbol{w}_{\boldsymbol{m e m}}^{\boldsymbol{b}}$ & $\boldsymbol{A}=\boldsymbol{a} \cdot \boldsymbol{w}_{\boldsymbol{m e m}}^{\boldsymbol{b}}$ \\
\hline $\mathbf{a}$ & -0.773 & $2.47 \cdot 10^{3}$ & $2.17 \cdot 10^{2}$ \\
\hline $\mathbf{b}$ & - & -4.35 & -3.45 \\
\hline $\mathbf{R}^{\mathbf{2}}$ & 0.977 & 0.998 & 0.998 \\
\hline $\mathbf{c}$ & 6.45 & - & - \\
\hline & Influence of $\boldsymbol{t}_{\boldsymbol{m e m}}$ for $w_{\text {mem }}$ & $=4 \mathrm{~mm}$ and $w_{\text {cross }}=0.8 \mathrm{~mm}$ \\
\hline & $\boldsymbol{S}_{\boldsymbol{x}}=\boldsymbol{a} \cdot \boldsymbol{t}_{\boldsymbol{m e m}}+\boldsymbol{c}$ & $\boldsymbol{S}_{\boldsymbol{x}}=\boldsymbol{a} \cdot \boldsymbol{t}_{\boldsymbol{m e m}}^{\boldsymbol{b}}$ & $\boldsymbol{A}=\boldsymbol{a} \cdot \boldsymbol{t}_{\boldsymbol{m e m}}^{\boldsymbol{b}}$ \\
\hline $\mathbf{a}$ & 18.6 & $3.88 \cdot 10^{3}$ & $7.18 \cdot 10^{2}$ \\
\hline $\mathbf{b}$ & - & 1.75 & 1.61 \\
\hline $\mathbf{R}$ & 0.997 & 0.984 & 0.983 \\
\hline $\mathbf{c}$ & 2.79 & - & - \\
\hline
\end{tabular}




\section{The influence of the foil geometries}

Figure 10 gives the stiffnesses and the anisotropies of a steel/Si suspension in dependence of (a.) $d_{f o i l}$ and $w_{\text {foil }}$ and of (b.) $d_{\text {foil }}$ and $t_{\text {foil }}$ (for $w_{\text {mem }}=4 \mathrm{~mm}$ ). For larger $d_{\text {foil }}$, lower stiffnesses and lower anisotropies can be obtained. Increasing $w_{\text {foil }}$ and $t_{\text {foil }}$ increase $S_{x}, S_{z}$ and $A$. Interestingly for the case of $t_{\text {foil }}=25 \mu \mathrm{m}$, A is increasing with $d_{f o i l}$, because at thinner $t_{f o i l}$, the influence of $d_{f o i l}$ on $S_{z}$ is reduced. Isotropic behavior can be obtained for $w_{\text {mem }}=5 \mathrm{~mm}$ with stiffnesses much lower than obtained for double Si suspensions.

Stiffness $\mathrm{S}_{\mathrm{x}}, \mathrm{S}_{\mathrm{z}}\left[\mathrm{N} \cdot \mathrm{mm}^{-1}\right] \quad$ Anisotropy A Stiffness $\mathrm{S}_{\mathrm{x}}, \mathrm{S}_{\mathrm{z}}\left[\mathrm{N} \cdot \mathrm{mm}^{-1}\right] \quad$ Anisotropy A
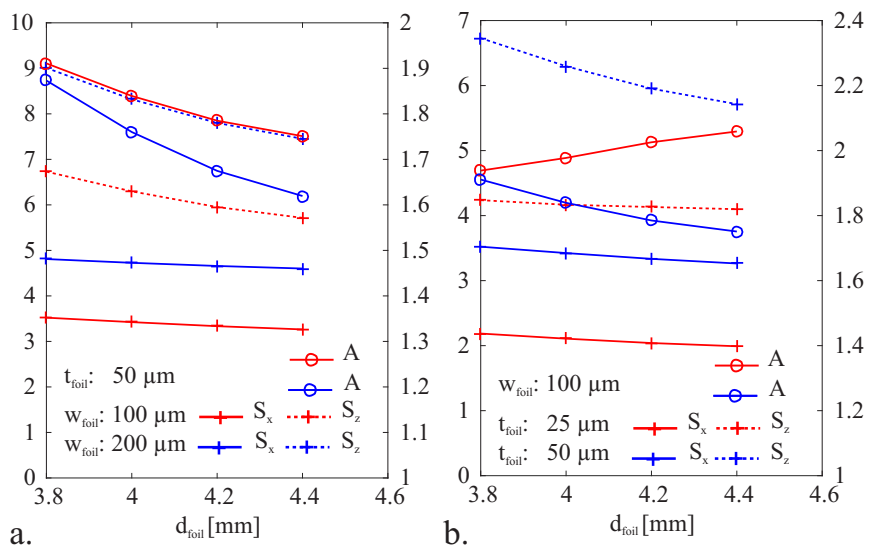

Figure 10 -Stiffnesses and anisotropies of a steel/Si suspension in dependence: of $a . d_{\text {foil }}$ and $w_{\text {foil }}$ and of $b . d_{\text {foil }}$ and $t_{\text {foil }}$ as obtained by simulations for $w_{\text {mem }}=4 \mathrm{~mm}$.

Note: $S_{x} / S_{z}$ and $A$ are plotted on different scales.

\section{Comparison of the three suspensions types}

In Figure 11, stiffnesses and anisotropies for single, double and steel/Si suspension are given as a function of $w_{m e m}$, which was identified earlier as the geometric parameter with the strongest influence.
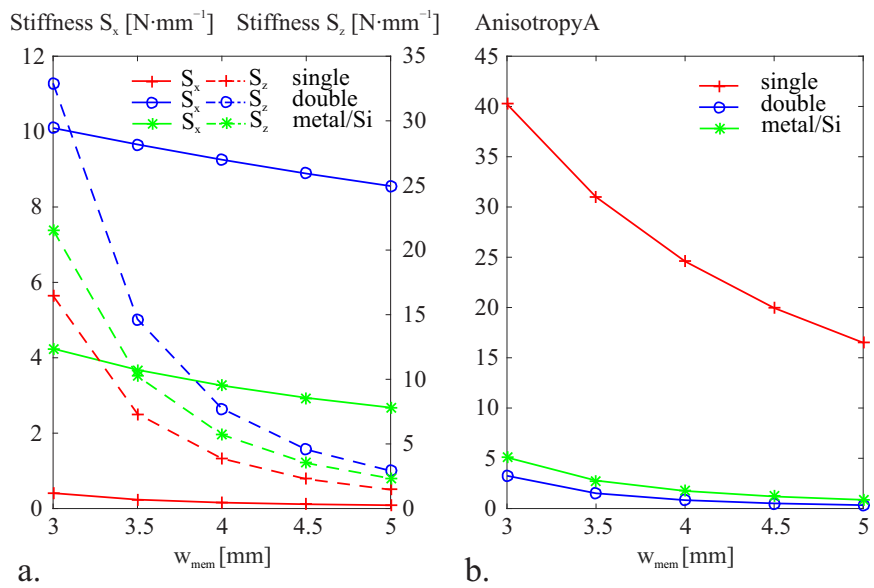

Figure 11 - Comparison of a. stiffnesses and $b$. anisotropies $A$ in dependence of $w_{\text {mem }}$ of the three suspensions types. Note: $S_{x} / S_{z}$ are plotted on different scales.

Through the use of stacked constructions, the stiffnesses $S_{x}$ and $S_{z}$ are influenced at very different strengths, which opens the window for anisotropy tailoring crossing the point of mechanical isotropy $(A=1)$. Compared to double $\mathrm{Si}$ suspensions, the stiffnesses of steel/Si suspensions are smaller.

\section{c. Design for manufacturing}

A subset of geometries was selected, by which the simulations should be verified with fabricated microprobes. The membranes were chosen to be either $w_{\text {mem }}=4$ or $5 \mathrm{~mm}$ wide. The other parameter variations realized in micro fabricated systems are listed in Table 5.

Table 5 - Designs for micro manufacturing.

\begin{tabular}{cccccc}
\hline $\begin{array}{c}\text { Design } \\
\text { single }\end{array}$ & $\begin{array}{c}\boldsymbol{w}_{\text {mem }} \\
{[\mathbf{m m}]}\end{array}$ & $\begin{array}{c}\boldsymbol{t}_{\text {mem }} \\
{[\boldsymbol{\mu m}]}\end{array}$ & $\begin{array}{c}\boldsymbol{w}_{\text {cross }} \\
{[\mathbf{m m}]}\end{array}$ & $\boldsymbol{A}$ \\
\hline S4 & 4 & 25 & 0.9 & & 24.5 \\
\hline S5 & 5 & 25 & 0.9 & & 16.4 \\
\hline & & & & & \\
\hline $\begin{array}{c}\text { Design } \\
\text { double/Si }\end{array}$ & $\begin{array}{c}\boldsymbol{w}_{\text {memB }} \\
{[\mathbf{m m}]}\end{array}$ & $\begin{array}{c}\boldsymbol{w}_{\text {mem }} \text { [ } \\
{[\mathbf{m m}]}\end{array}$ & $\begin{array}{c}\boldsymbol{t}_{\text {mem }} \\
{[\boldsymbol{\mu m}]}\end{array}$ & $\begin{array}{c}\boldsymbol{w}_{\text {cross }} \\
{[\mathbf{m m}]}\end{array}$ & $\boldsymbol{A}$ \\
\hline D4 & 4 & 4 & 25 & 0.9 & 0.922 \\
\hline D5 & 5 & 5 & 25 & 0.9 & 0.187 \\
\hline D45 & 4 & 5 & 25 & 0.7 & 0.553 \\
\hline
\end{tabular}

\begin{tabular}{ccccccc}
\hline $\begin{array}{c}\text { Design } \\
\text { steel/Si }\end{array}$ & $\begin{array}{c}\boldsymbol{w}_{\text {mem }} \\
{[\mathbf{m m}]}\end{array}$ & $\begin{array}{c}\boldsymbol{d}_{\text {foil }} \\
{[\mathbf{m m}]}\end{array}$ & $\begin{array}{c}\boldsymbol{w}_{\text {foil }} \\
{[\mathbf{m m}]}\end{array}$ & $\begin{array}{c}\boldsymbol{t}_{\text {mem }} \\
{[\boldsymbol{\mu m}]}\end{array}$ & $\begin{array}{c}\boldsymbol{w}_{\text {cross }} \\
{[\mathbf{m m}]}\end{array}$ & $\boldsymbol{A}$ \\
\hline MS4_19 & 4 & 4 & 0.1 & 25 & 0.9 & 1.95 \\
\hline MS4_29 & 4 & 4.4 & 0.2 & 25 & 0.9 & 1.69 \\
\hline MS4_27 & 4 & 4.4 & 0.2 & 25 & 0.7 & 1.87 \\
\hline MS5_19 & 5 & 4.3 & 0.1 & 25 & 0.9 & 1.08 \\
\hline MS5_29 & 5 & 4.9 & 0.2 & 25 & 0.9 & 0.91 \\
\hline MS5_17 & 5 & 4.3 & 0.1 & 25 & 0.7 & 1.00 \\
\hline
\end{tabular}

\section{MANUfaCturing PROCESS}

\section{a. Si-cross membrane}

The silicon membranes are micro fabricated out of a doublesided, polished (100)-silicon wafer with a thickness of $360 \pm 25 \mu \mathrm{m}$. The integrated sensors are piezo-resistors, which are realized in the silicon by the use of a double boron doping and wired by aluminum tracks. Finally, the membrane is etched in $40 \% \mathrm{KOH}$ by a temperature of $80^{\circ} \mathrm{C}$. Double layers of silicon dioxide $\left(\mathrm{SiO}_{2}\right)$ and silicon nitride $\left(\mathrm{Si}_{3} \mathrm{~N}_{4}\right)$ are used as a mask during the wet etching. The process is stopped when the desired membrane thickness is reached (Figure 12a.). During the process, the thickness is monitored using a stylus profiler (Dektak 8, Co. Veeco Metrology Group) in order to predict the required time of etching. In doing so, a thickness tolerance of about $\pm 3 \mu \mathrm{m}$ can be reached. $[1,2]$ 


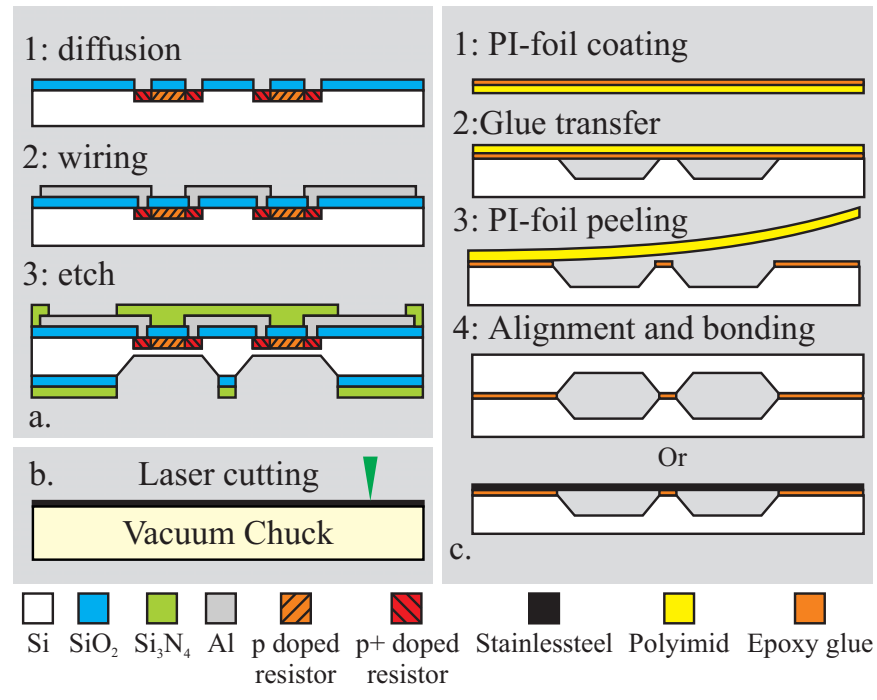

Figure $12-a$. The fabrication process of silicon membrane with integrated piezo sensors [1, 2], $b$. fs-laser structuring of stainless steel foil and c. bonding technique with a special transfer process.

\section{b. Metal foil suspension}

$50 \mu \mathrm{m}$ thick stainless steel foils (material: X5CrNi18-10 or AISI 304) were micro machined in a maskless ablation process by means of a femtosecond laser (microSTRUCTc, 3D Micromac AG equipped with a YB:KGW solid state laser, Pharos, Light Conversion) at $515 \mathrm{~nm}$ wavelength (frequency doubled). An F-Theta lens with a $100 \mathrm{~mm}$ focal length and a galvanometer scanner (Scanlab RTC5) are used to position the laser beam with a high processing speed. The influence of scan speed and pulse energy was investigated in a cutting test matrix. At a pulse frequency of $200 \mathrm{kHz}$, a scan speed of $1250 \mathrm{~mm} / \mathrm{s}$ and a pulse energy of $205 \mu \mathrm{J}$, the foils are efficiently cut and appeared without any thermal deformation. Contours with a $50 \mu \mathrm{m}$ width were cut using a $6 \mu \mathrm{m}$ equidistant lines as laser spots leading strategy. 55 repetitions of the strategy enable a proper cutting of the $50 \mu \mathrm{m}$ thick foil. To maintain the foil in position during the cutting a porous ceramic vacuum chuck is used (Figure 12b.) allowing to process complete 4" wafer sizes (Figure 13) in about 2 hours. The cut contours are cleaned by plunging foils for about $30 \mathrm{~s}$ in a $\mathrm{FeCl}_{3}$ solution $(4.0 \mathrm{M})$, by which all ablation induced particles disappear. To clean the foil surface from every organic residue which can affect the quality of the adhesive bonding a piranha solution $\left(\mathrm{H}_{2} \mathrm{SO}_{4}\right.$ and $\mathrm{H}_{2} \mathrm{O}_{2}$ at 3:1) was compared with a simple acetone/ethanol cleaning and the contact angles with distilled water were measured. For the simple cleaning, an angle of $71^{\circ} \pm 3^{\circ}$ was found whereas after piranha cleaning an angle of $23^{\circ} \pm 2^{\circ}$ could be measured. However, this strong wettability is not permanent and the bonding has to happen within a couple of hours.

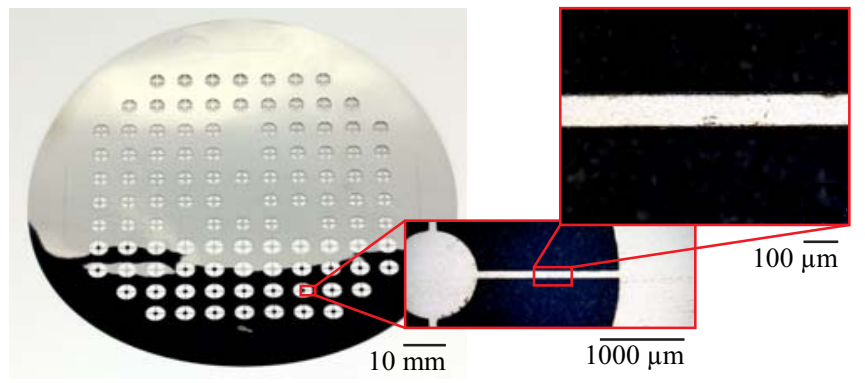

Figure 13 - Result of structured 4" foil by fs-laser and details of the rod.

\section{c. Bonding process}

For both stacked designs epoxy adhesive (353 ND, Co. Epotek) was used for the bonding technique described in Figure 12c.

The adhesive is spin coated on a $50 \mu \mathrm{m}$ thick and Ø4" Polyimide-foil (PI-foil), which is temporarily applied on a PDMS coated glass wafer. The PI-foil is first cleaned with acetone and ethanol in a spinning machine. After mixing the resin with hardener at weight proportion of 10:1 and after 20 min degassing, $2 \mathrm{~mL}$ of high viscosity adhesive is spun on the PI-foil first over $30 \mathrm{~s}$ at a rotation speed of $500 \mathrm{~min}^{-1}$, during which the glue is spread over $70 \%$ of the foil surface and in the second step over $30 \mathrm{~s}$ at $4000 \mathrm{~min}^{-1}$ to get the appropriate distribution over the complete PI-foil. After the PI-foil is peeled off its sticky side is temporarily applied to the bottom silicon wafer while taking care that no air bubbles are trapped in between. After the PI-foil is removed a thin and homogeneous adhesive layer remains on the silicon substrate which now is directly bonded to the top substrate.

Both substrates are aligned with a double-sided alignment system (EVG®620, EVG ${ }^{\circledR} 420$ from EV Group). During the alignment process, the silicon top wafer is held by vacuum fixation at the rim. In the case of the stainless steel suspensions, the metal foil is temporarily laminated on a PI foil coated with thin PDMS-layer in order to maintain a planar form. The epoxy adhesive is cured on a hotplate at $150^{\circ} \mathrm{C}$ for $2 \mathrm{~min}$. The resulting stack is temporarily bonded with resist on a dummy wafer to protect sensors during the sawing process. Single sensor chips are cleaned in acetone and ethanol. Figure 14 shows bonded wafers, single chips and cross section views of the adhesive interfaces, which illustrate perfect alignment and bonding qualities. 

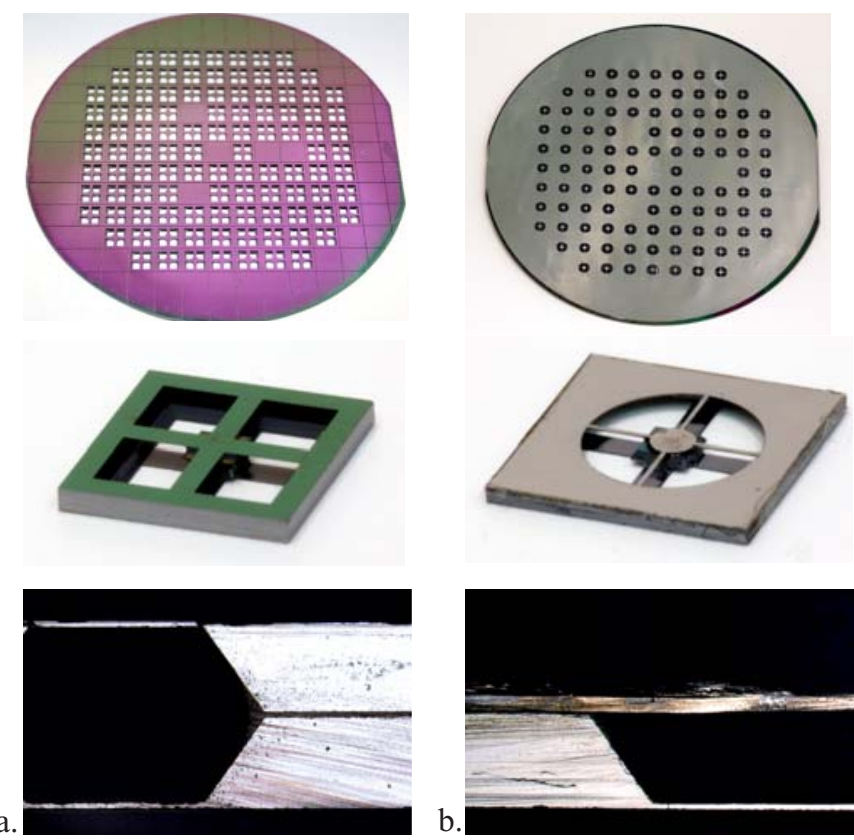

Figure 14 - Bonding results of a. double Si suspension crossmembranes and b. steel/Si suspension. Top: after wafer level bonding, middle: after chip cutting; bottom: cross sectional views (obtained by sawing) of the bond interface.

\section{d. Final montage of microprobe before characterization}

Each stylus was fabricated with geometries described in Chapter III by eroding a tungsten carbide electrode with a $0.2 \mathrm{~mm}$ diameter wire on a $\mu$ EDM-machine (SARIX SX200-HPM equipped with an SX-Micro-Fine-Pulse-ShapeGenerator and an SX-Arianna wire unit,) [2]. The stylus was manually glued on the middle of the sensor with the epoxy adhesive already used for previous bonding. In Figure 14, fully mounted single, double and steel/Si-membrane microprobes are shown. For the first mechanical characterization, these sensors were fixed on a PCB with adhesive and electrically contacted with conductive glue (Delo Dualbond IC343, Co. Delo) by a flip chip process to enable readout of the four Wheatstone bridges [1].

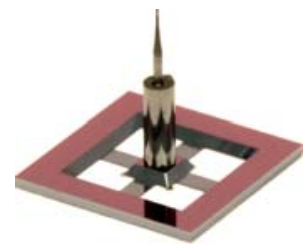

a.

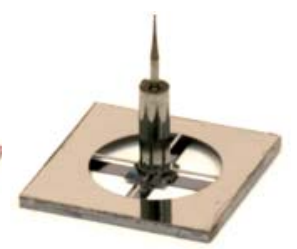

b.

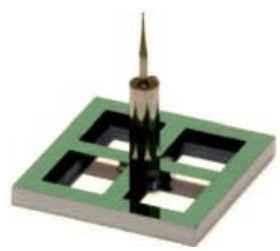

c.
Figure 15 - Sensor chips with a mounted stylus for a. single Si-, b. steel/Si - and c. double Si suspension.

\section{RESULTS AND ANALYSIS}

For measuring the stiffnesses of the microprobes, a setup with two linear stages controlled by a LabVIEW ${ }^{\circledR}$ program was used to deflect the probe in a defined direction. A motor stage (M-531.5iM from PI GmbH with a resolution of $0.1 \mu \mathrm{m}$ and a large stroke of $306 \mathrm{~mm}$ ) has been used for the contact detection with the microprobe. A piezo actuator (P-841.60 from PI $\mathrm{GmbH}$ ) with a resolution of $1.8 \mathrm{~nm}$ and a $90 \mu \mathrm{m}$ stroke has been used for the deflection of the tip [22, 23]. For probing the other directions, the microprobe can be rotated. A calibrated load cell (KD78 from ME-Meßsysteme $\mathrm{GmbH}$ ) allows measuring forces within a range of $500 \pm 500 \mathrm{mN}$ with an accuracy class of $0.1 \%$. The absolute deflection of the probing system is given by the stage motion and the deflection of the load cell. The load cell was calibrated as described earlier.[1]

In Figure 16, representative force-deflection diagrams for two different designs with negligible non-linearities and with no hysteresis are presented, from which the stiffnesses were obtained by linear regression. The use of additional materials other than silicon (epoxy glue and stainless steel) can result in internal stresses at elevated temperatures or in the presence of temperature gradients. But the use of this system is foreseen in a temperature controlled metrology laboratory where appropriate measurement uncertainties can be obtained. Already during the bonding process at elevated temperatures, stresses can be introduced. They could be reduced by cold bonding or by implementing two-step (lower temperature pre-curing followed by higher temperature curing) bonding processes. Despite these concerns, a perfectly linear response of the systems without hysteresis can be observed as it can be seen in Figure 16.
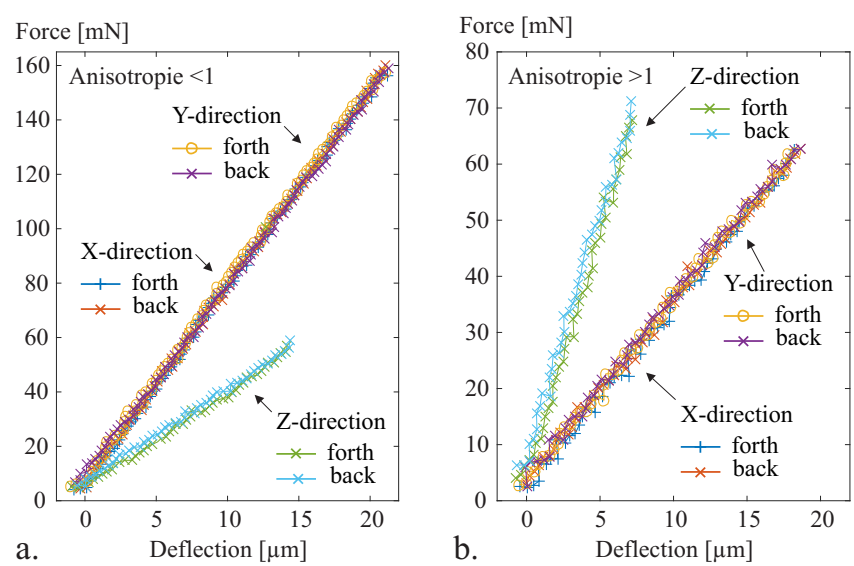

Figure 16 - Force diagram for the designs: a. double Si suspension D45 and b. steel/Si suspension MS4_29.

Figure 17 shows the experimentally obtained stiffnesses for two single $\mathrm{Si}$ suspensions in comparison to results of the previous simulation, which are in good agreement. The indicated tolerances of measured values correspond to the interval of confidence for 3 measurements and an alpha $=0.01$. 
Stiffness $\mathrm{S}_{\mathrm{x}}\left[\mathrm{N} \cdot \mathrm{mm}^{-1}\right] \quad$ Stiffness $\mathrm{S}_{\mathrm{z}}\left[\mathrm{N} \cdot \mathrm{mm}^{-1}\right] \quad$ Anisotropy A

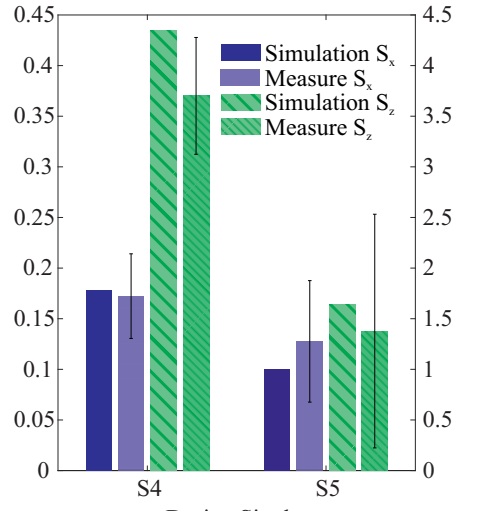

a. Design Single

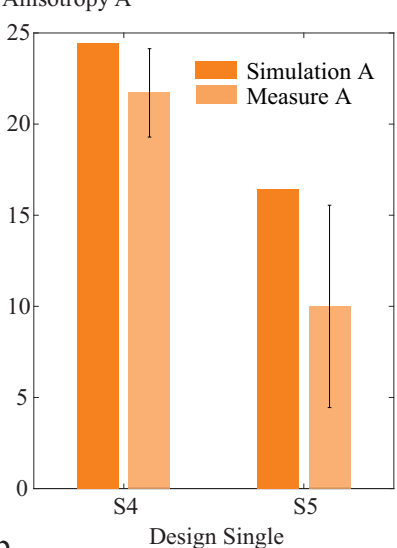

b.

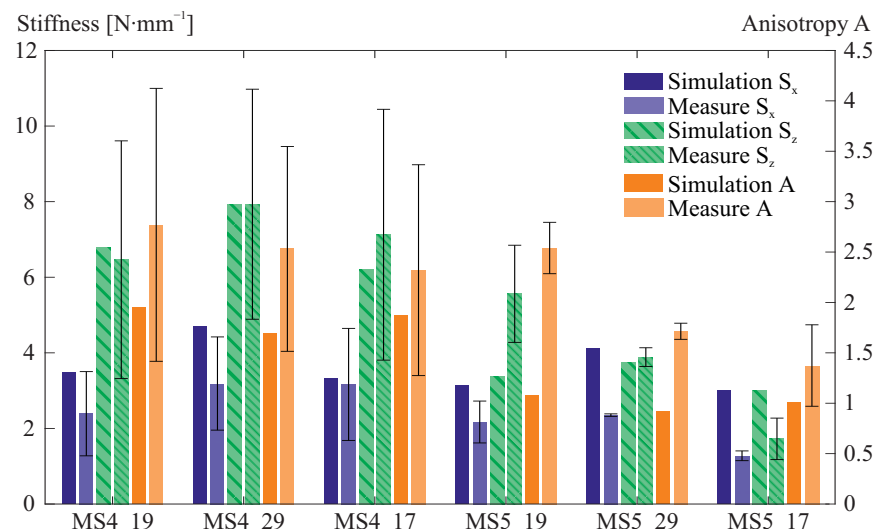

Design steel/Si

Figure 17 - Simulation vs. measuring results for single Si suspension: $a$. stiffness in $x$-z direction and $b$. anisotropy. Note: $S_{x} / S_{z}$ are plotted on different scales.

Figure 18 shows the equivalent comparison for the double $\mathrm{Si}$ suspension which does not show significant deviations.

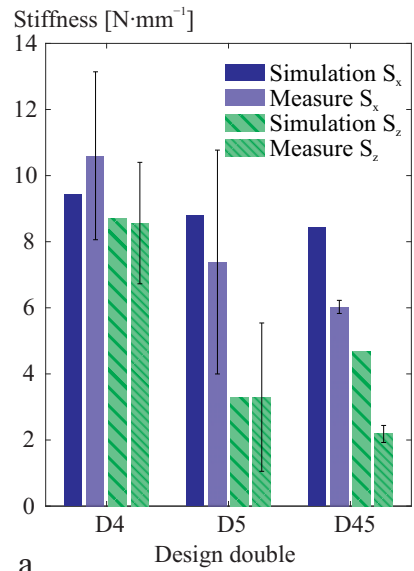

a.

Figure 18 - Simulation vs. measuring results for double Si suspension designs: $a$. stiffness in $x-z$ direction and $b$. anisotropy. Note: $S_{x} / S_{z}$ are plotted on different scales.

Figure 19 shows equivalent results for the steel/Si suspension. In general, the simulated values were reached in experiments.

The deviations of experimental results from simulations assuming ideal geometries can be explained by non-ideal system realizations. The thickness of the Si-membrane can vary up $\pm 2 \mu \mathrm{m}$ over the same wafer. For the double-Si suspension, such errors can add up in the worst case. The use of SOI-Wafer would result in more homogeneous membrane thickness. Also, the fabrication and the mounting of the stylus can create non-ideal geometries. Further, during the bonding of both substrates misalignment and stress resulting from CTE mismatch can be induced. Through optimized production and automated stylus mounting such deviations could be minimized.

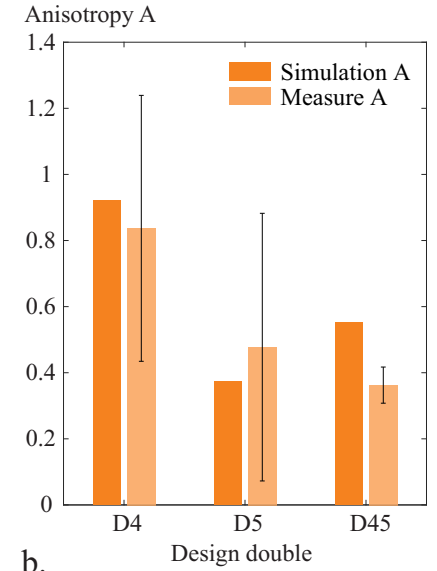

\footnotetext{
/ $/ \mathrm{Si}$
} 
values of the obtained sensitivities can be explained by reduced deformations occurring by stacked suspensions. Nevertheless, the obtained sensitivities are good enough to measure with submicron precision.

Table 7 - Comparison of sensitivities $E_{x}$ and $E_{z}$ between measured and from literature values.

\begin{tabular}{|c|c|c|c|}
\hline Designs & $\begin{array}{c}\text { Single 3x3 } \\
{[1]}\end{array}$ & $\begin{array}{l}\text { Steel/Si 4x4 } \\
\text { Figure 20a. }\end{array}$ & $\begin{array}{l}\text { Double 4x4 } \\
\text { Figure 20b. }\end{array}$ \\
\hline$E_{x}\left[\mathrm{mV} \cdot \mathrm{V}^{-1} \cdot \mu \mathrm{m}^{-1}\right]$ & $3.2 \pm 0.2$ & 0.620 & 0.330 \\
\hline$E_{z}\left[\mathrm{mV} \cdot \mathrm{V}^{-1} \cdot \mu \mathrm{m}^{-1}\right]$ & $20.4 \pm 0.4$ & 10.2 & 8.83 \\
\hline
\end{tabular}

\section{CONCLUSION AND OUTLOOK}

New stacked microprobe designs were investigated in simulations, fabricated with aid of wafer level bonding processes, and characterized in experiments. In particular, a new bonding technique with a special adhesive transfer technique was developed. The characterization of the prototypes shows only minor differences between simulations and measurements, which can result from fabrication tolerances. Our results confirm that microprobes with stacked suspensions can be produced with tailored anisotropies (between 3 and 0.4). The stylus of systems, which anisotropies are lower than 1 , could be replaced by a longer stylus to obtain an isotropic behavior. A longer stylus is advantageous when probing structures with higher aspect ratios. With piezoresistive transducers, good sensitivities can be achieved for dimensional measurement with a sub-micron precision. This new design of microprobes is intended to be integrated into conventional CMMs for further $3 \mathrm{D}$ calibrations and artifact measurements. With lower anisotropies, the measurement uncertainties can reduce. Despite the achieved improvements, the measurement range still has to be increased in order to enable easier integration and longer usage. Future work will, therefore, include investigations with thinner metal foils and thinner Simembranes, for instance by the use of SOI wafers, in order to get isotropic systems with further reduced stiffnesses and improved measurement range.

\section{ACKNOWLEDGMENT}

This research was supported by the Deutsche Forschungsgemeinschaft, DFG, grant number DI 1934/5-1. We thank I. Ekinci, E. Charfi, and Z. Bingxiang for assistance during the development, fabrication, and measurements.

\section{REFERENCES}

[1] Ferreira, N., Brennecke, A., Dietzel, A., Buttgenbach, S., Krah, T., Metz, D., Kniel, K., Hartig, F., "Reducing the probe ball diameters of 3D siliconbased microprobes for dimensional metrology", 2013 Seventh International Conference on Sensing
Technology (ICST), Wellington, New Zealand, pp. 301-306, 2013. DOI 10.1109/ICSensT.2013.6727663.

[2] Ferreira, N., "Entwicklung, Integration und Erprobung eines piezoresistiven 3D-Mikrotasters", Shaker, Aachen, 2015. ISBN 9783844035469.

[3] Weckenmann, A., Peggs, G., Hoffmann, J., "Probing systems for dimensional micro- and nano-metrology", Measurement Science and Technology, vol. 17, no. 3, pp. 504-509, 2006. DOI 10.1088/0957-0233/17/3/S08.

[4] Weckenmann, A., Estler, T., Peggs, G., McMurtry, D., "Probing Systems in Dimensional Metrology", CIRP Annals - Manufacturing Technology, vol. 53, no. 2, pp. 657-684, 2004. DOI 10.1016/S0007-8506(07)60034-1.

[5] Goj, B., Dressler, L., Hoffmann, M., "Design and characterization of a resonant triaxial microprobe", Journal of Micromechanics and Microengineering, vol. 25 , no. 12 , p. 125011,2015 . DOI 10.1088/09601317/25/12/125011.

[6] Schwenke, H., Wäldele, F., Weiskirch, C., Kunzmann, H., "Opto-tactile Sensor for 2D and 3D Measurement of Small Structures on Coordinate Measuring Machines", CIRP Annals - Manufacturing Technology, vol. 50, no. 1, pp. 361-364, 2001. DOI 10.1016/S0007-8506(07)621404.

[7] Neuschaefer-Rube U.; Bremer H.; Hopp B.; Christoph R., "Recent developments of the 3D fiber probe", 11th Laser Metrology for Precision Measurement and Inspection in Industry (2014), Tsukuba, Japan.

[8] “", Optische Messtechnik Stein GmbH, URL: http://www.optische-messtechnik.ch/deutsch/. Accessed on 19.01.2017.

[9] Küng, A., Meli, F., Thalmann, R., "Ultraprecision microCMM using a low force 3D touch probe", Measurement Science and Technology, vol. 18, no. 2, pp. 319-327, 2007.

[10]Alblalaihid, K., Lawes, S., Kinnell, P., "Variable stiffness probing systems for micro-coordinate measuring machines", Precision Engineering, vol. 43, pp. 262-269, 2016. DOI 10.1016/j.precisioneng.2015.08.004.

[11]Peggs, G. N., Lewis, A. J., Oldfield, S., "Design for a Compact High-Accuracy CMM", CIRP Annals Manufacturing Technology, vol. 48, no. 1, pp. 417-420, 1999. DOI 10.1016/S0007-8506(07)63216-8.

[12]Widdershoven, I., Donker, R. L., Spaan, H. A. M., "Realization and calibration of the "Isara 400" ultraprecision CMM", Journal of Physics: Conference Series, vol. 311, p. 12002, 2011. DOI 10.1088/1742$6596 / 311 / 1 / 012002$.

[13]Bos, E. J.C., "Tactile 3D probing system for measuring MEMS with nanometer uncertainty:aspects of probing, design, manufacturing and assembly", Technische Universiteit Eindhoven, 2008. ISBN 978-90-386-1216-4.

[14]Bos, E.J.C., Heldens, R.W.P., Delbressine, F.L.M., Schellekens, P.H.J., Dietzel, A., "Compensation of the anisotropic behavior of single crystalline silicon in a 3D 
tactile sensor", Sensors and Actuators A: Physical, vol. 134 , no. 2 , pp. 374-381, 2007. DOI

10.1016/j.sna.2006.05.043.

[15]Ferreira, N., Krah, T., Jeong, D. C., Metz, D., Kniel, K., Dietzel, A., Büttgenbach, S., Härtig, F., "Integration of a silicon-based microprobe into a gear measuring instrument for accurate measurement of micro gears", Measurement Science and Technology, vol. 25, no. 6, p. 64016, 2014. DOI 10.1088/0957-0233/25/6/064016.

[16]Buetefisch, S., Dai, G., Danzebrink, H.-U., Koenders, L., Solzbacher, F., Orthner, M. P., "Novel design for an ultra high precision 3D microprobe for CMM applications", Procedia Engineering, vol. 5, pp. 705-712, 2010. DOI 10.1016/j.proeng.2010.09.207.

[17]Bauccio, M., "ASM metals reference book", 9. ed., ASM International, Materials Park, OH, 2005. ISBN 9780871704788.

[18]Gere, J. M., "Mechanics of Materials", 6. ed., Brooks/Cole-Thomson Learning Inc., 2004. ISBN 0534-41793-0.

[19]"Handbook of refractory carbides and nitrides, Properties, characteristics, processing, and applications", Pierson, H. O. (editor), Westwood, NJ, 1996. ISBN 9780815513926.

[20]Wortman, J. J., Evans, R. A., "Young's Modulus, Shear Modulus, and Poisson's Ratio in Silicon and Germanium", Journal of Applied Physics, vol. 36, no. 1, pp. 153-156, 1965. DOI 10.1063/1.1713863.

[21]Malhaire, C., "Caractérisation et modélisation de microtransducteurs de pression à hautes performances intégrés sur silicium.”, These, L'institut national des sciences appliquées de lyon, Lyon, 1998.

[22]“M-5x1.5i”, Yumpu, URL: https://www.yumpu.com/en/document/view/25338 696/micropositioners-hexapod-systems-pi-physikinstrumente/45. Accessed on 13.07.2017.

[23]"PI - Solution for precision motion and positioning", Physik Instrumente (PI) GmbH, URL: https://www.physikinstrumente.com/. Accessed on 13.07.2017. 